\title{
Urgent Multivisceral Resection due to Perforated Primary Gastric Lymphoma
}

\author{
Jurij Janež
}

Department of Abdominal Surgery, Universitiy Medical Centre Ljubljana, Zaloška Cesta 7, 1525 Ljubljana, Slovenia, Europe.

\section{Corresponding Author:}

Dr. Jurij Janež

Email: jurij.janez@gmail.com

This is an Open Access article distributed under the terms of the Creative Commons Attribution License (creativecommons.org/ licenses/by/3.0).

Received

Accepted

Published

June 13, 2017

August 13, 2017

September 15, 2017

\begin{abstract}
Background: Multivisceral resection is feasible option for advanced gastric cancer and is indicated, when radical resection with curative intent can be achieved. It is not the best option for palliative treatment, because it has many possible complications. Case Report: We report a case of a 38-year-old male patient, referred with radiologic signs of pneumo-peritoneum and large gastric tumor with suspected perforation. Abdominal computed tomography showed pneumo-peritoneum and gastric tumor mass with suspected perforation. Distal subtotal gastrectomy with lymp node dissection, distal subtotal pancreatectomy and segmental resection of colon transversum with end to end colo-colic anastomosis was performed. Histopathological examination revealed a large B-cell lymphoma, diffuse type, with high proliferative index Ki-67 (nearly 100\%). Conclusion: Multivisceral resection is a feasible option in cases of perforated primary gastric lymphoma, especially when radical resection can be achieved.
\end{abstract}

Keywords: Gastrectomy, Lymphoma, Pancreatectomy, Spontaneous Perforation, Stomach Neoplasms.

\section{Introduction}

Perforation of the stomach is a common complication of peptic ulcer disease, however it is rarely associated with gastric malignancy [1]. Primary gastric lymphoma is rare, accounting for only $1 \%-5 \%$ of all gastric tumors [2]. A spontaneous perforation has been reported from $1 \%$ to $5 \%$ of cases of primary gastric lymphoma and commonly occurs after chemotherapy and irradiation [3]. Emergency surgery is usually performed but the prognosis in cases of perforated gastrointestinal lymphoma is very poor [4]. This report describes a case of a young man with spontaneous perforation of primary gastric malignant lymphoma and diffuse peritonitis without prior chemotherapy.

\section{Case Report}

A38-year old man was transferred to our department from other clinic because of acute abdomen.
Emergency abdominal computed tomograpy (CT) was performed, which showed a large gastric tumor mass with suspected perforation on anterior gastric wall and free intraperitoneal air [Fig.1]. The patient was affected with severe abdominal pain, hypotension, tachycardia and leukocytosis. Abdominal wall was board-like rigid with rebound tenderness. We decided for an emergeny operation. Prior to the emergency operation, upper intestinal endoscopy was performed, which showed a large tumor mass in distal half of the stomach, suspected to be a gastric adenocarcinoma. Median laparotomy was performed. During abdominal exploration we found a large gastric tumor, encompassing distal two third of the stomach. On the anterior gastric wall there was a large perforation measuring approximately $6 \mathrm{~cm}$ in diameter. Free intraperitoneal fluid was present with signs of diffuse peritonitis. There was no other signs of intraabdominal malignancy. The gastric tumor was infiltrating into the pancreatic body and transverse 
mesocolon. We performed distal subtotal gastrectomy with Billroth II reconstruction, distal subtotal pancreatectomy and segmental resection of transverse colon. After the resection and intestinal reconstruction was completed, we made an extensive abdominal irrigation with saline, inserted two abdominal drains and closed the abdominal wall.

After the last operation patient had some respiratory problems and pleural effusions, which were managed with pleural drainage and invasive respiratory support. In the postoperative course, the patient developed a minor gastroenteral fistula from gastroenteral anastomosis, which was seen on gastrointestinal contrast study and a minor pancreatic fistula, proved with elevated levels of amylase and lipase in the abdominal drain fluid. Both fistulas were successfully managed conservatively. Further postoperative course was uneventful.

Final histopathological examination revealed a diffuse, large B-cell lymhoma, with high proliferative index Ki-67 (nearly 100\%). Lymhoid nature of these cells was indicated by strongly positive immunohistochemical reaction staining for CD10, CD20, MUM1, myc, bcl-2 and bcl-6. Two perigastric lymph nodes were also infiltrated with diffuse large B-cell lymphoma cells, and eight perigastric lypmh nodes were directly infiltrated with gastric tumor. Distal (duodenal) resection margin was without tumor cells, but proximal resection margin was focally infiltrated with lymphoma tumor cells. There was no tumor in the resected pancreas, only signs of chronic atrophic pancreatitis. The resected segment of transverse colon was also without tumor.

Patient was presented to oncologic multidisciplinary team, that indicated postoperative chemotherapy, as soon as the patient will be enough rehabilitated. Few days later the patient was referred to oncology for further treatment.

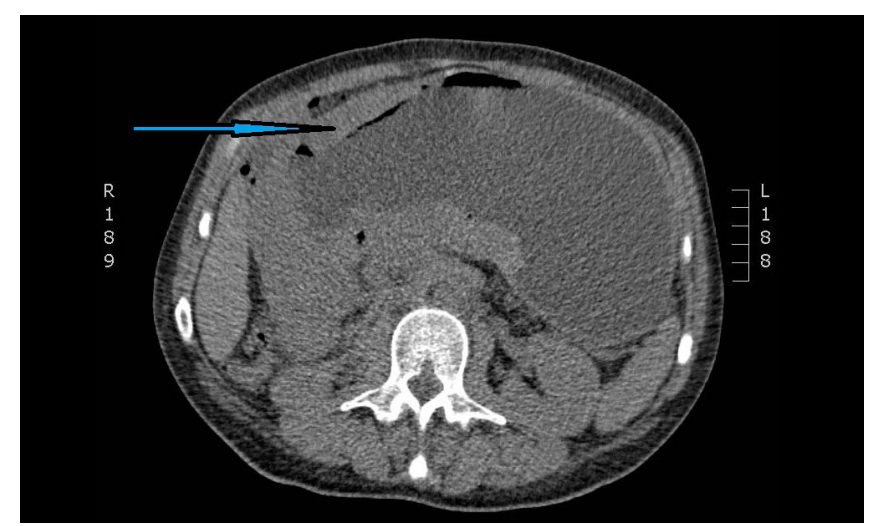

Fig.1: Abdominal computed tomography showes enlarged stomach with tumor mass in the distal half of the stomach with thick gastric wall (arrow).

\section{Discussion}

Gastrointestinal lymphoma represents a heterogenous group with differences in pathogenesis, treatment and prognosis. Stomach is the most common site of gastrointestinal lymphoma and the second most common gastric lymphoma is diffuse large B-cell lymphoma (DLBCL) [5]. Spontaneous perforation of primary gastric lymphoma without prior chemotherapy is rare, only few have been described in literature. Perforation of the malignant gastric lymphoma during chemotherapy is a well-known event, however the incidence is not high. Gastric lyphoma is associated with minimal desmoplastic reaction; therefore, spontaneous perforation occurs more frequently in this disease than in gastric carcinoma [6].

It is difficult to preoperatively diagnose primary gastric lymphoma with spontaneous perforation. At our hospital, emergency endoscopic examination is not routinely performed for patients suspected of having gastroduodenal perforation. In those patients we perform a diagnostic laparoscopy or exploratory laparotomy. In our patient, a perforated gastric tumor was found at emergency abdominal exploratory laparotomy. At that time, we did not know, that the tumor is lymphoma. We suspected, that it is more likely a gastric 
adenocarcinoma. We had to perform a multivisceral resection, because tumor was infiltrating into the pancreas and transverse mesocolon. Final histopathological examination revealed, that the tumor is a primary gastric diffuse large B-cell lymphoma (DLBCL).

Modern treatment of primary gastric DLBCL consists of chemotherapy and radiation to provide a curative outcome [7]. Standard combination chemotherapy consists of R-CHOP (rituximab plus cyclophosphamide, doxorubicin, vincristine, prednisone). Those patients, who are Helicobacter pylori positive, may be treated with antibiotics alone. Perforation is rarely seen with chemotherapy, but remains a risk in fewer than 5\% of patients. Primary surgical debulking is no longer considered standard of care and surgical resection has a limited value in the treatment of gastric lymphoma [8].

Khadraoui and colleagues reported a case of gastric perforation in advanced stage of DLBCL, which happened during the first cycle of chemotherapy, but was successfully treated conservatively with percutaneous endoscopic gastrostomy for gastric drainage and percutaneous endoscopic jejunostomy for feeding, without surgery during ongoing chemotherapy. Authors concluded, that in proper settings gastric resection can be avoided even in the emergent event of perforation [8].

Multivisceral resection can present in the postoperative course with minor and major complications. Our patient had respiratory distress and pleural effusions, which were managed with pleural drainage and invasive respiratory support. In the postoperative course we observed a minor gastric and pancreatic fistula, both were successfully managed conservatively. Shoji and colleagues reported a case of a 65-year old man with gastroduodenal malignant lymphoma (diffuse large B-cell type) accompanied with a bulbar perforation. They performed a pancreaticoduodenectomy because the duodenal second portion and pancreatic head were also involved. The postoperative course was uneventful and chemotherapy was effective in eliminating the involved lymph nodes. Authors concluded, that the choice of surgical resection is very important in patients with perforated gastric lymphoma, since chemotherapy should be started as soon as possible after surgery [4].

\section{Conclusion}

Gastric lymphoma is a rare and enigmatic disease. All patients with a histologically confirmed gastric lymphoma should be discussed at a multidisciplinary conference. Standard treatment for a parimary gastric lymphoma is a chemoradiotherapy. Surgery should be reserved only for complications, such as hemorrhage, perforation or obstruction. It is difficult to preoperatively diagnose primary gastric lymphoma with spontaneous perforation. In the proper settings even a perforated gastric lymphoma can be managed conservatively, without operation.

Contributors: JJ: manuscript writing, literature search, surgery and final approval of the manuscript. JJ will act as guarantor.

Funding: None; Competing interests: None stated.

\section{References}

1. Yabuki K, Tamasaki Y, Satoh K, Maekawa T, Matsumoto M. Primary gastric lymphoma with spontaneous perforation: report of a case. Surg Today. 2000;30:10301033.

2. Ohkura Y, Lee S, Kaji D, Ota Y, Haruta S, Takeji Y, et al. Spontaneous perforation of primary gastric malignant lymphoma: a case report and review of the literature. World Journal of Surgical Oncology. 2015;13:35.

3. Aroori S, Matthews S, Collum N, Carey PD. Primary gastric non-Hodgkiń s lymphoma: 13 yearś experience. Irish journal of Medical Science. 2002;171:23-33.

4. Shoji T, Konno H, Tanaka T, Baba M, Kamiya K, Kaneko $\mathrm{T}$, et al. Pancreaticoduodenectomy for perforated gastric malignant lymphoma: report of a case. Surg Today. 2002;32:468-471.

5. Fischbach W. Gastric MALT lymphoma - Update on diagnosis and treatment. Best Practice \& Reserch Clinical Gastroenterology. 2014;28:1069-1077.

6. Mittal B, Wasserman TH, Griffith RC. Non-Hodgkińs lymphoma of the stomach. Am J Gastroenterol. 1983;78:780-787. 
7. Lopez-Zemundio J, Ramirez-Gonzalez LR, NunezMarquez J, Fuentes Orozco C, Gonzalez Ojeda A, Leonher-Ruezga KL. Gastric perforation by MALT lymphoma. Case report. Cir Cir. 2015;83:217-221.

8. Khadraoui H, Feigin KN, Fox, JJ, Ong L, Shike M, Yahalom $\mathrm{J}$, et al. Successful management of gastric perforation in stage IV diffuse large B-cell lymphoma with chemoradiation therapy, percutaneous endoscopy gastrostomy for gastric drainage, and percutaneous endoscopy jejunostomy for nutrition. Clinical Lymphoma, Myeloma \& Leukemia. 2013;13:327-330. 